\title{
Dawn of High Energy Spin Physics — In Memory of Michel Borghini
}

\author{
Akira Masaike \\ Professor Emeritus of Kyoto University \\ 4-3-14 Aoyamadai, Abiko-shi, \\ Chiba-ken, 270-1175, Japan \\ masaike@mvb.biglobe.ne.jp \\ Published 29 February 2016
}

\begin{abstract}
High energy spin physics with the polarized proton target in 1960s is shown. The dynamic polarization in which the electronic polarization is transferred to protons in paramagnetic material by means of magnetic coupling was proposed at the beginning of 1960s. The first $\mathrm{N}-\mathrm{N}$ experiment using a polarized proton target was performed with the crystal of $\mathrm{La}_{2} \mathrm{Mg}_{3}\left(\mathrm{NO}_{3}\right)_{12} 24 \mathrm{H}_{2} \mathrm{O}$ at CEN-Saclay and Berkeley in 1962, followed by $\pi$-p experiments in several laboratories. Protons in organic materials were found to be polarized up to $80 \%$ in ${ }^{3} \mathrm{He}$ cryostats in 1969. It was helpful for large background experiments. High proton polarization was interpreted in the spin temperature theory. Spin frozen targets were constructed in early 1970s and used for experiments which require wide access angle. Michel Borghini was a main player for almost all the above works.
\end{abstract}

\section{Introduction}

It is a great honor for me to give a talk on the dawn of high energy spin physics in memory of Michel Borghini, who played an important role in progress of high energy spin physics. His contribution in this field has been highly appreciated.

M. Borghini was born in 1934. After graduation from Ecole Polytechnique in Paris in 1955, he joined the group of Anatole Abragam at CEN-Saclay. He started his academic career in studying the nuclear magnetic resonance in Saclay, in particular dynamic polarization of protons, which was applied to the wide field of particle physics. He devoted his full effort to the development of the polarized proton target, with which he made a significant contribution to high energy physics throughout 1960s.

M. Borghini opened a new field in particle physics, 'high energy spin physics', dawn of which I will briefly look at.

This is an Open Access article published by World Scientific Publishing Company. It is distributed under the terms of the Creative Commons Attribution 3.0 (CC-BY) License. Further distribution of this work is permitted, provided the original work is properly cited. 


\section{A. Masaike}

\section{Dynamic Polarization of Protons}

High energy spin physics began in early 1960s. It was pointed out that studying the spin dependent forces is one of the most important issues for particle physics. Therefore, it became an urgent need to measure the spin parameters of particle reactions. ${ }^{1}$ In order to realize such experiments, it was indispensable to polarize the target protons.

The dynamic method which transfers the electronic polarization to protons using magnetic couplings between electronic and nuclear spins was proposed in France and the US.

Nuclei with magnetic moments in thermal equilibrium at the temperature $T$ and magnetic field $B$ could be oriented to the direction of field. The degree of nuclear polarization $P_{n}$ with a spin $I=1 / 2$ is given by

$$
P_{n}=\tanh (\mu B / k T),
$$

where $B$ and $\mu$ are the external magnetic field and the nuclear magnetic moment, respectively. It is

$$
P_{n}=\tanh \left(1.02 \times 10^{-7} B / T\right)
$$

for protons. In principle, the proton polarization of $76 \%$ could be obtained at $0.01 \mathrm{~K}$ and in $10 \mathrm{~T}$. However, it is quite difficult to realize such high polarization. On the other hand, we can get sizable polarizations of nuclei in paramagnetic materials by means of the dynamical method in rather lower field and at higher temperature. In the dynamical method magnetic coupling between electron spins and nuclear spins is used to transfer the polarization of electrons to nuclei. The method was proposed by Abragam for paramagnetic materials. ${ }^{2}$ The phenomenon was named 'solid effect'.

The energy level diagram of a paramagnetic center coupled to a single neighboring proton in high external magnetic field is shown in Fig. 1(a). The transition $a$ in Fig. 1(a) is a 'forbidden transition' in which both $S_{z}$ and $I_{z}$ reverse the sign. While the transition $b$ in which $S_{z}$ reverses and $I_{z}$ remains the same are called 'allowed transition'. The dipole-dipole coupling does ensure that there is a small admixture of $S_{z}=+1 / 2$ and $-1 / 2$. The admixture makes forbidden transitions possible by RF field which is applied at the frequency corresponding to the transition $a$.

The populations of the two levels connected by the forbidden transition can be equalized. It corresponds to the "flip-flop" of an electron spin and a proton spin as shown in Fig. 1(b). Because of the strong coupling between electrons and lattice the relaxation time of electron $\left(T_{1 s}\right)$ is short, whereas, the proton spin stays on the level for long time since coupling between the proton spin and the lattice is weak. As two neighboring protons are coupled by the dipole-dipole interaction, the orientation of proton spin diffuses throughout whole material, trending to equalize the nuclear polarization. 


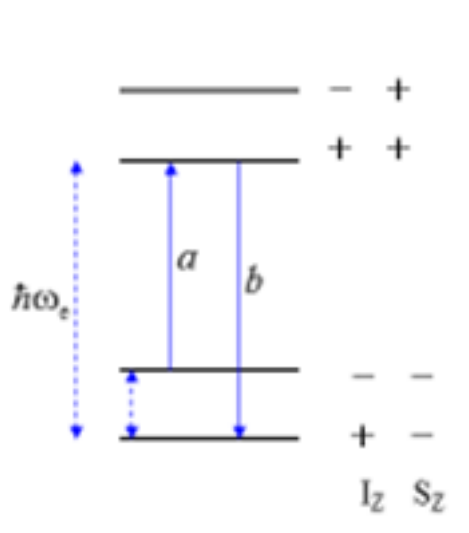

(a)
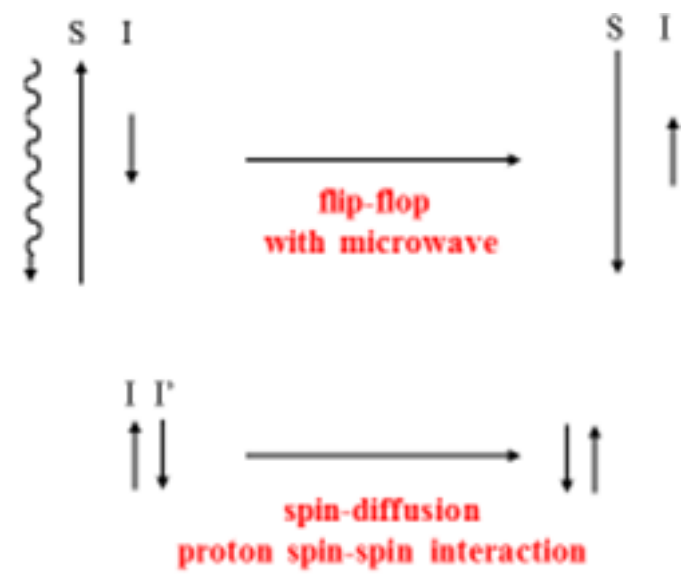

(b)

Fig. 1. (a) Energy level diagram of the paramagnetic center coupled to the proton. (b) Flip-flop of an electron spin and a proton spin by means of microwave irradiation. The nuclear spin diffusion is also shown.

At the beginning of the history of dynamically polarized target, protons in the crystal of $\mathrm{La}_{2} \mathrm{Mg}_{3}\left(\mathrm{NO}_{3}\right)_{12} 24 \mathrm{H}_{2} \mathrm{O}$, (LMN), containing a small percent (e.g. $0.2 \%$ ) of $\mathrm{Nd}$ and Ce, were polarized by Abragam ${ }^{3}$ and $\mathrm{Jeffries}^{4}$, respectively.

Since the NMR signals for positive and negative polarizations are well-resolved for LMN, the solid effect works so well. The proton polarization of about $80 \%$ was obtained in the temperature around $1 \mathrm{~K}$ and the magnetic field of $1.8 \mathrm{~T}$. The LMN targets were successfully operated for elastic scattering experiments with $\pi, \mathrm{K}, \mathrm{p}$ and $n$ beams.

\section{High Energy Physics with Polarized Target in 1960s}

The success of the dynamic polarization was amazing-event for high energy physicists, since it promised a new field of particle physics. At the International Conference on Polarized Targets held at Saclay in 1966, Dalitz pointed out that the polarized target may lead to especially illuminating information on three major areas in particle physics.

(i) High energy scattering where Regge-pole exchange is dominant.

(ii) Tests of time-reversal invariance for electromagnetic processes.

(iii) Hadron spectroscopy. Many resonant states had been observed for mesonic and baryonic states. In the attempt to classify and understand these hadronic states, the first need is for the determination of the spin and the parity for each state.

The first experiment with the polarized target was performed to measure the parameter $\mathrm{C}_{\mathrm{nn}}$ for p-p scattering at $20 \mathrm{MeV}$ by Abragam, Borghini, Catillon, 


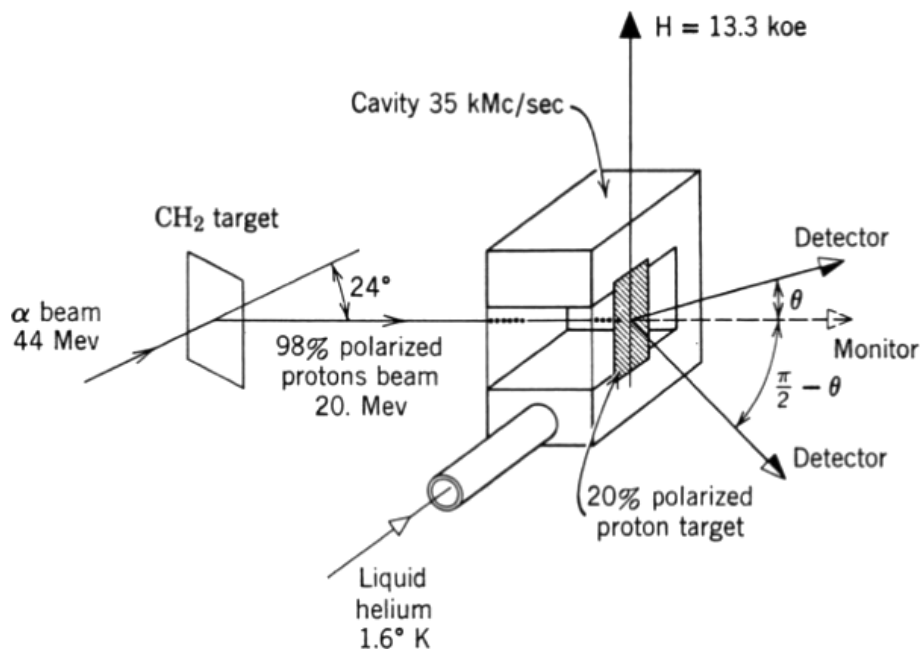

Fig. 2. Apparatus of a p-p scattering experiment with a polarized target at Saclay.

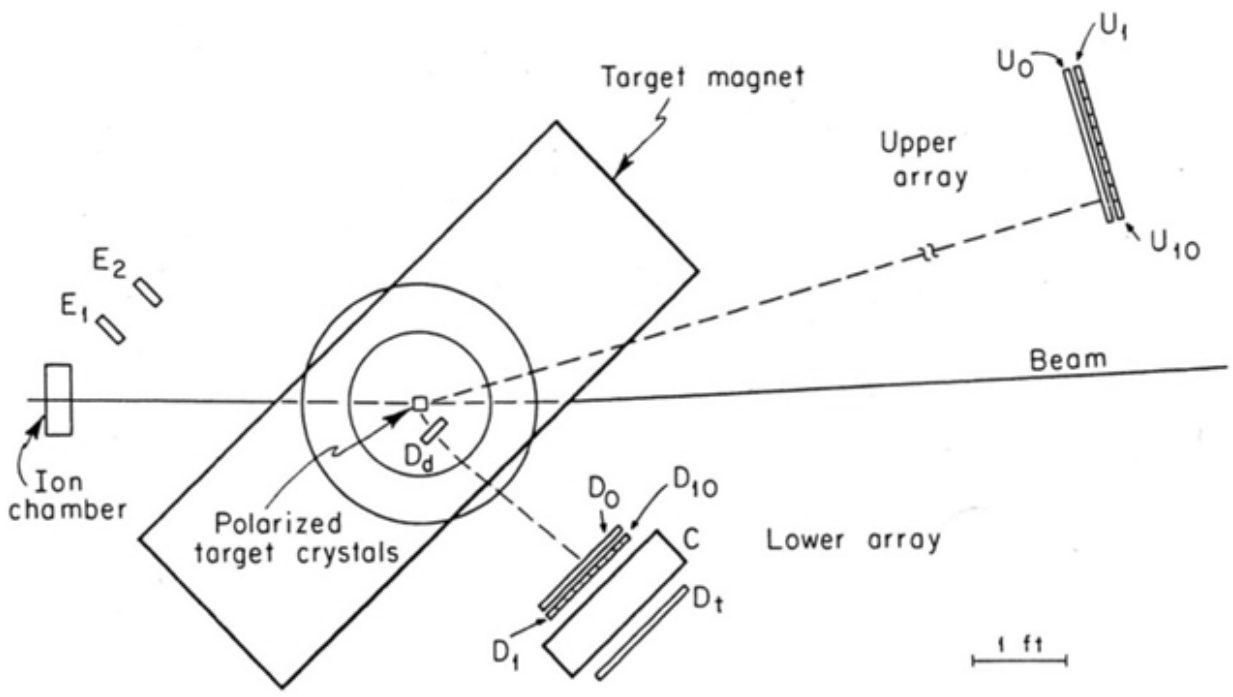

Fig. 3. Experimental setup for $\pi$-p scattering at Berkeley.

Coustham, Roubeau and Thirion at Saclay in $1962^{5}$. The experiment was done with a polarized beam on a polarized protons in LMN.

The first pion-proton scattering experiment was performed at Berkeley (Bevatron) by Chamberlain, Jeffries, Schutz, Shapiro, and van Rossum in $1963 .{ }^{6}$ In this experiment it was necessary to measure the both angles of pion and proton in order to check the coplanarity, since the background from complex nuclei were enormous. 
$10 \mathrm{GeV} / \mathrm{c}$

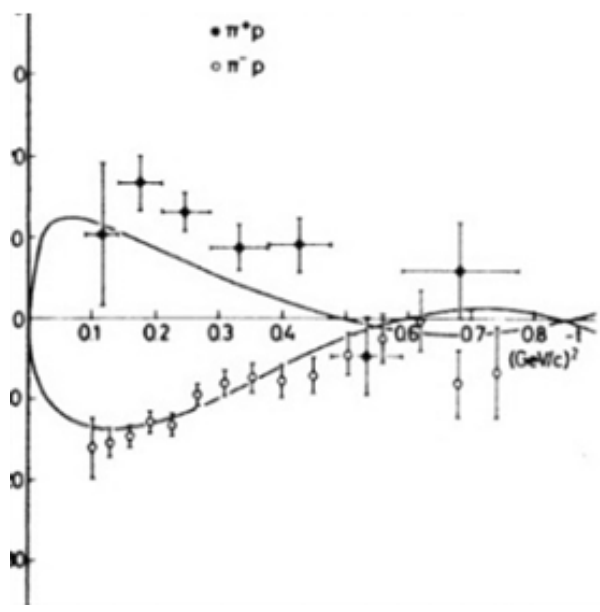

$12 \mathrm{GeV} / \mathrm{c}$

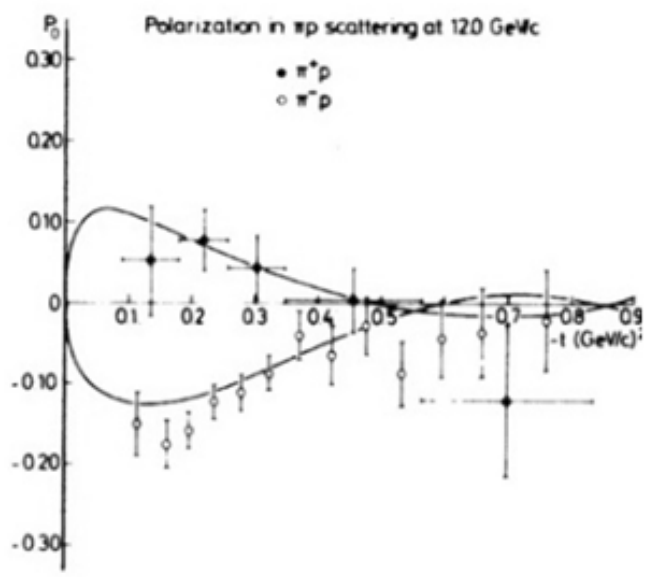

Fig. 4. Asymmetries of pion-proton elastic scattering measured at CERN.

In 1965, a pion-proton scattering experiment was made with $\sim 1 \mathrm{GeV}$ pions by Atkinson, Cox, Duke, Heard, Jones, Kemp, Murphy, Prentice and Thresher at Rutherford. ${ }^{7}$ It indicated three nucleon resonances, that is, $\mathrm{N}^{*}(1674) 5 / 2$, $\mathrm{N}^{*}(1688) 5 / 2^{+}, \mathrm{N}^{*}(1920) 7 / 2^{+}$, which had not been previously seen.

A pion-proton scattering experiment was also made with $\sim 2 \mathrm{GeV}$ pions by Suwa, Yokosawa Booth, Esterling and Hill at Argonne in 1965. ${ }^{8}$ The result showed a higher nucleon resonance, $\mathrm{N}^{*}(2190) 7 / 2$-.

Asymmetries of pion-proton elastic scattering were measured at $10-12 \mathrm{GeV} / \mathrm{c}$ by Dick, Borghini et al. at CERN. ${ }^{1}$ The results showed a mirror symmetry for $\pi^{-} \mathrm{p}$ and $\pi^{+}$p scatterings.

The LMN targets were successfully operated for these experiments. And the polarized target became a very important tool for particle physics.

In the mid-1960s LMN polarized targets were constructed in many high energy laboratories, including Berkeley, Argonne, Los Alamos and Harvard in the USA, Saclay and Grenoble in France, Rutherford and Liverpool in UK, Nagoya-INS in Japan, Dubna and Protvino in USSR, and CERN. No polarized target was constructed in SLAC and DESY, because radiation damages of LMN crystal with electrons and $\gamma$-beams were serious.

\section{Organic Materials for Polarized Target}

Although LMN was an excellent polarized target material for elastic scattering experiments, it was not convenient for other experiments, e.g. backward scattering, inelastic scattering, rare decay etc. because of the enormous amount of background 


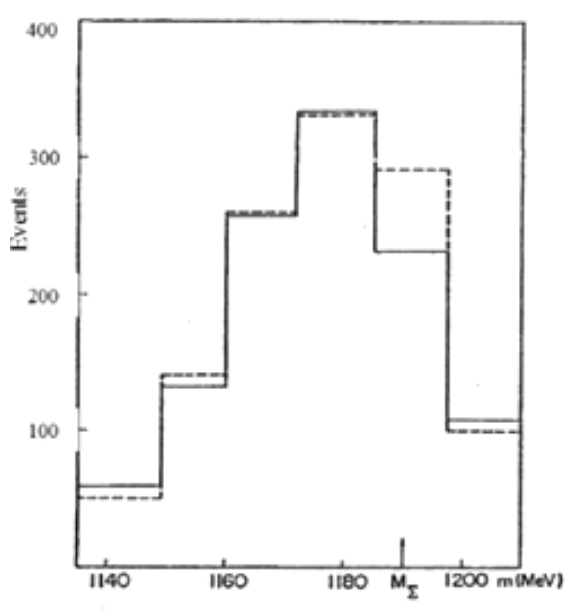

(a)

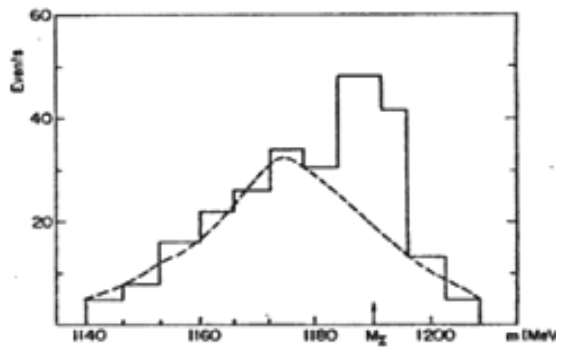

(b)

Fig. 5. Missing mass spectra of $\sum$ with (a) LMN and (b) $\mathrm{CH}_{2}$ targets ${ }^{9}$.

events related to nuclei different from hydrogen, as the dilution factor (polarizable nucleons/total number of nucleons) is too small. Asymmetry measurements of $\pi^{+}+$ $\mathrm{p} \rightarrow \mathrm{K}^{+}+\Sigma^{+}$reaction were tried to carry out at CERN and Berkeley in order to test the parity conservation in strong interaction using LMN targets, it was difficult to identify the events from free protons, since the ratio of free protons to bound protons was $1: 15$.

Furthermore, it was difficult to use LMN targets for electron and photon beams, since crystal of LMN is damaged seriously with relativistic particles of $2 \times 10^{12} / \mathrm{cm}^{2}$. Therefore, the dynamic polarization of protons in organic materials was tested with free radicals in the last half of $1960 \mathrm{~s}$, since these materials have higher concentration of free protons, and are 250 times stronger for radiation damage. More than 200 kinds of organic materials with several sorts of free radicals had been tested to polarize by Borghini et al. at CERN. Despite the tremendous efforts had been made, none of materials had been successfully polarized up to $25 \%$ before 1968 .

Breakthroughs were achieved dramatically in 1969. Protons in butanol with small amount of water doped with porphyrexide were polarized up to $40 \%$ at $1 \mathrm{~K}$ and in 2.5 $\mathrm{T}$ by Mango, Runolfsson and Borghini at CERN. ${ }^{10}$ At the same time protons in diol with $\mathrm{Cr}^{5+}$ complex were polarized up to $45 \%$ at $1 \mathrm{~K}$ in $2.5 \mathrm{~T}$ by Glättli et al. at Saclay. ${ }^{11} \mathrm{~A}$ few months later protons in diol were found to be polarized up to $80 \%$ at the temperature lower than $0.5 \mathrm{~K}$ by Masaike et al. at Saclay, ${ }^{12}$ while Hill et al. at Argonne succeeded in polarizing protons in butanol up to $67 \%$ in a ${ }^{3} \mathrm{He}$ cryostat. ${ }^{13}$ These values are surprisingly large, since it had been believed before their trials that the polarization at lower temperature than 1 $\mathrm{K}$ would be less than that at $1 \mathrm{~K}$. Then, polarized targets with diols and butanols cooled in ${ }^{3} \mathrm{He}$ cryostats have been used in most of high energy physics laboratories. 


\section{Spin Temperature Theory}

Abragam and Redfield proposed the spin temperature theory in which a spin system isolated from the lattice and subjected to spin-spin interactions proceeds toward an equilibrium such that the probabilities of finding the system on the energy levels are given by a Boltzmann distribution, $\exp \left(-E_{i} / k T_{s}\right)$, where $T$ is the spin temperature of the state. ${ }^{14}$ In this hypothesis, spin systems behave in the same way as the systems considered in thermodynamics.

The spin temperature theory was applied to dynamic nuclear polarization by Solomon (Saclay) in 1962 following a work of Goldman and Landesman on two nuclear species. ${ }^{15}$ They pointed out that the electron non-Zeeman reservoir is in close thermal contact with the nuclear Zeeman reservoir through thermal mixing, and their common spin temperature evolves towards high nuclear polarization.

Borghini reinvented the spin temperature theory for dynamic nuclear polarization in $1968^{16}$ and introduced it to particle physicists at Berkeley Conference on Polarized Target in $1971 .{ }^{9}$ He showed a model of spin packets for the inhomogeneous broadening of the electronic resonance lines, with which he computed the nuclear polarization under microwave irradiation in low temperature and named the spin temperature model the 'Donkey Effect'.

Borghini claimed that more than two electrons participate to the dynamic polarization in this model.

\section{6. ${ }^{3} \mathrm{He}-{ }^{4} \mathrm{He}$ Dilution Refrigerators for Spin Frozen Target}

In 1965 Schmugge and Jeffries discussed the possibility of maintaining the polarization without microwave irradiation, if the nuclear spin relaxation time is long enough. ${ }^{17}$ Such a target is advantageous because of the large access angle around

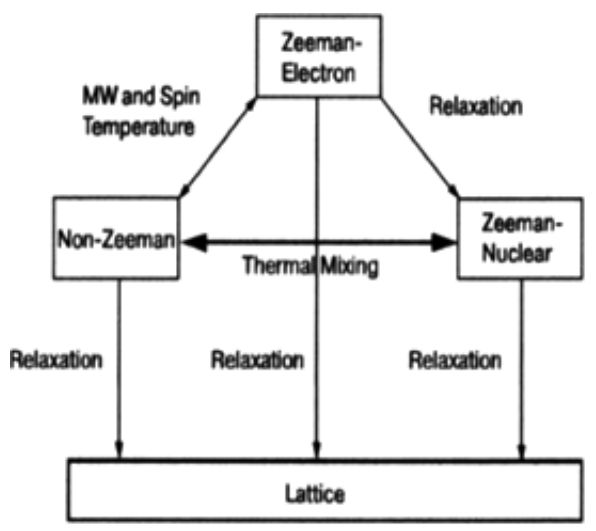

(a)

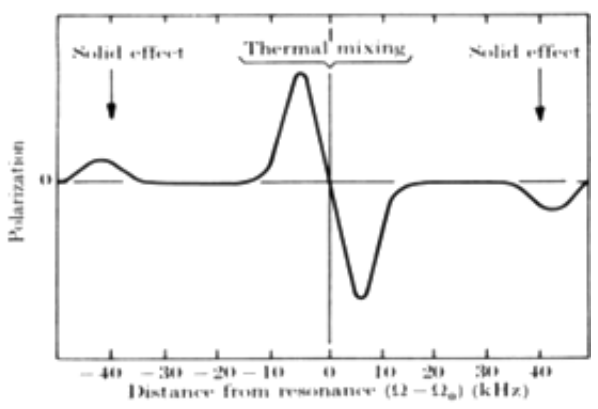

(b)

Fig. 6. (a) Thermal couplings between 4 thermal reservoirs: electronic Zeeman, electronic nonZeeman, nuclear Zeeman and lattice in the course of DNP under microwave irradiation ${ }^{15}$. (b) NMR signals enhanced by sold effect and thermal mixing ${ }^{14}$. 


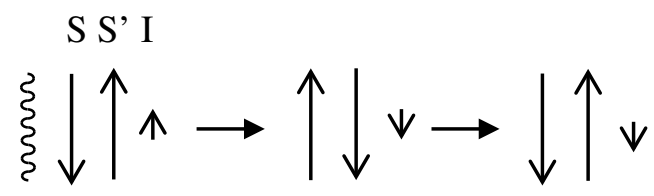

Fig. 7. Cooling of the nuclear spin by the electron spin-spin interaction reservoir. ${ }^{9}$

the target area in less homogeneous and lower magnetic field than in the polarizing condition.

${ }^{3} \mathrm{He}-{ }^{4} \mathrm{He}$ dilution refrigerators were developed toward higher polarization in lower temperature than 0.1K. Neganov (Dubna) and Varoquaux (Orsay) pointed out the possibilities to use the ${ }^{3} \mathrm{He}^{-}{ }^{4} \mathrm{He}$ dilution refrigerator for the polarized target in $1966 .^{1}$

Nagamine (Tokyo) made a dilution refrigerator for static polarization of Bi nuclei in $1969 .^{9}$

The first spin frozen target with a horizontal dilution refrigerator was realized by Niinikoski in 1971 and was operated successfully at CERN in $1974 .^{18}$

Soon after the success at CERN, a spin frozen deuteron target was constructed at KEK for the reactions $\mathrm{K}^{+} \mathrm{n} \rightarrow \mathrm{K}^{+} \mathrm{n}, \mathrm{K}^{0} \mathrm{p} .{ }^{19}$ Then, Saclay group constructed a spin frozen target in late 1970 s for nucleon-nucleon scattering experiments. ${ }^{20} \mathrm{It}$ consisted of a high power dilution refrigerator for target material of $100 \mathrm{~cm}^{3}$, a vertical polarizing magnet and two holding magnets. Spin frozen targets were also constructed in Bonn, PSI, Dubna and other laboratories.

\section{Neutron Polarization with Proton Filter}

Neutron transmitted through polarized protons are polarized, since neutrons with spin anti-parallel to the proton spin are scattered away. L. Shapiro at Dubna proposed to make a polarized slow neutron beam using an LMN filter in $1966 .{ }^{1}$ Ishimoto et al. made a polarized neutron beam using an ethylene glycol filter at KEK in $1976 .{ }^{21}$ The method was used for parity violation experiments with polarized neutrons of $0.02 \sim 1 \mathrm{eV}$ at Dubna, KEK and Los Alamos in 1980s.

\section{Concluding Remarks}

The polarized proton target has been playing a significant role in particle and nuclear physics for the last 50 years.

We would stress that Michel Borghini made tremendous contributions to the progress of 'High Energy Spin Physics' through polarizing the proton in 1960s.

We wish to honor the life and work of Michel Borghini.

\section{References}

1. in Proceedings of the International Conference on Polarized Targets and Ion Sources, Saclay, France 1966 (ed. by CEN-Saclay). 
2. A. Abragam, Phys. Rev. 98, 1729 (1955).

3. A. Abragam et al. Phys. Lett. 2, 310 (1963).

4. T. J. Schmugge, C. D. Jeffries, Phys. Rev. Lett, 9, 268 (1963).

5. A. Abragam et al. Phys. Lett. 2, 310 (1962).

6. O. Chamberlain et al. Phys. Lett. 7, 293 (1963).

7. H. H. Aktinson et al. Proc. Roy. Soc. A289, 449 (1966).

8. S. Suwa et al.Phys. Rev. Lett. 15560 (1965).

9. in Proceedings of the 2nd International Conferece on Polarized Targets (Berkeley 1971).

10. S. Mango, O. Runolfsson and M. Borghini, Nucl. Instr. Meth. 72, 45 (1969).

11. H. Glättli et al. Phys. Lett. 29A, 250 (1969).

12. A. Masaike et al. Phys. Lett. 30A, 63 (1969).

13. D. Hill et al. Phys. Rev. Lett. 23, 460 (1969).

14. M. Goldman, Spin Temperature and Nuclear Magnetic Resonance in Solid (Oxford University Press 1970).

15. I. Solomon, in XI Colloque Ampere on Magnetic and Electric Resonance and Relaxation, p.25 (1962) Eindhoven, Netherland (North-Holland, Amsterdam).

16. M. Goldman and A. Landesman, Phys. Rev. 132, 610 (1963).

17. M. Borghini, Phys. Rev. Lett. 20, 419 (1968).

18. T. J. Schmugge, C. D. Jeffries, Phys. Rev. 138A, 1785 (1965).

19. T. Niinikoski, F. Udo, Nucl. Instr. Meth. 134, 219 (1976).

20. S. Isagawa et al. Nucl. Instr. Meth. 154, 213 (1978).

21. J. Deregel et al. inProc. Int. Conf. on High Energy Physics with Pol. Beam and Targets, p. 463 (1980) Lausanne.

22. S. Ishimoto et al. Japanese Jour. Appl. Phys. 251246 (1986). 